\title{
VARIABLES QUE PREDICEN LA APARICIÓN DE SOBRECARGA EN CUIDADORES PRIMARIOS INFORMALES DE NIÑOS CON CÁNCER
}

\author{
VARIABLES THAT PREDICT THE APPEARANCE OF BURDEN IN INFORMAL PRIMARY \\ CAREGIVERS OF CHILDREN WITH CANCER
}

Xolyanetzin Montero Pardo', Samuel Jurado Cárdenas ${ }^{2}$ y José Méndez Venegas ${ }^{3}$

\author{
Universidad Autónoma de Sinaloa, México \\ Universidad Nacional Autónoma de México \\ Instituto Nacional de Pediatría. México
}

\section{Abstract}

The purpose of the study was to identify the psychological characteristics and care variables that predict the onset of primary informal caregivers burden (CPI) of children with cancer, in a hospital in Mexico City. The nonrandom sample consisted of 100 participants, who answered an integrated battery conformed by a health interview for the $\mathrm{CPI}$, the load scale for the Zarit caregiver, the anxiety and depression of Beck inventory, the family support for the CPI scale and the scale modes of coping with stress by Lazarus and Folkman. The results confirmed that women react as $\mathrm{CPI}$ to children with cancer, and in most cases the patient's mother. The variables of burden model stepwise multiple regressions were depression $\left(R^{2}=0.228\right)$, the number of affected areas in the caregiver's life $\left(R^{2}=0.319\right)$ and anxiety $\left(R^{2}=0.348\right)$. In all the three variables explained $34 \%$ of variance. While coping style, family support, time to patient care, daily care hours and the number of activities carried out were not significant variables in predicting overload. The findings of this study could be useful for designing interventions that promote physical, social and emotional wellbeing of this population, preventing the patient from becoming caregivers.

Keywords: Children with cancer, caregivers, burden, anxiety and depression.

Palabras clave: Niños con cáncer, cuidadores, carga, ansiedad y depresión.

\section{Correspondencia:}




\section{INTRODUCCIÓN}

La detección de una enfermedad crónica como el cáncer, en uno de los miembros de la familia, acarrea un cambio radical en el interior del individuo y su entorno. El cambio en el paciente está dado fundamentalmente por el sufrimiento y la limitación de las capacidades del enfermo, que requiere ser atendido, cuidado y medicado; mientras que el cambio en el entorno ocurre por la incorporación del equipo médico tratante y del sistema hospitalario ${ }^{(1-3)}$.

La enfermedad crónica de un niño produce cambios inevitables en los recursos familiares: emocionales, organizacionales, financieros y adaptativos ${ }^{(4,5)}$. Para la familia el cáncer significa una crisis y un cambio en su estilo de vida, ya que a las demandas familiares se le agrega la atención del paciente $^{(6-8)}$. Para ello, se designa implícita o explícitamente a un miembro de la familia como cuidador; este es aquella persona que, aunque no pertenece al equipo de salud institucional, ni se ha observado como tal, tiene la máxima responsabilidad en el cuidado y atención en casa del paciente dependiente o con discapacidad ${ }^{(9-11)}$.

Los cuidadores se clasifican como formales e informales ${ }^{(12)}$. Los cuidadores informales, se dividen en primarios y secundarios, la diferencia básica entre estos es el nivel de responsabilidades que tienen hacia el paciente y el tiempo que dedican a cuidarlo ${ }^{(13)}$. En este contexto, el cuidador primario es quien brinda apoyo a personas dependientes, para subsanar las necesidades no cubiertas por el sector formal, generalmente son familiares o amigos, no reciben retribución económica por la ayuda que ofrecen, los motiva un componente emocional, no disponen de capacitación específica y ofrecen atención sin límite de horario $^{(12)}$.

Cuidar de un familiar enfermo se asume ante la sociedad como parte de las
Ilamadas "tareas domésticas" y como tal, se asocia a un determinado rol de género: "es cosa de mujeres" ${ }^{\prime \prime 13,11)}$.

Las actividades que realizan los cuidadores son muy amplias y varían en relación con las características y necesidades del paciente. En el caso de los niños con enfermedades crónicas, se requiere una mayor vigilancia y disposición permanente de tiempo comparado con lo que implica cuidar de un niño sano ${ }^{(14-16)}$.

El cuidado de un familiar es una experiencia prolongada que exige reorganizar la vida familiar, laboral y social en función de las tareas que implica cuidar. Estas circunstancias, influyen de forma distinta dependiendo de las características, problemas o enfermedades que padece la persona que recibe los cuidados, de lo avanzada que esté la enfermedad, de la lucidez psíquica que posea y de lo autónomo que el niño/a sea ${ }^{(17,18)}$.

La participación del cuidador informal se considera fundamental en el campo de la salud. Sin embargo, se le presta poca o ninguna atención a los riesgos e implicaciones que conlleva esta actividad en la salud y bienestar de los cuidadores ${ }^{(13)}$. En muchas ocasiones, a lo largo del proceso de la enfermedad del paciente o familiar, el cuidador puede llegar a cargarse, agotarse y colapsarse, ante las demandas del cuidado, sus características propias y las de su contexto ${ }^{(19)}$.

Los cuidadores son personas vulnerables, ya que el compromiso de cuidar tiene generalmente importantes costos materiales, emocionales y de salud por dedicar su vida a cuidar a su familiar de forma intensa y continua ${ }^{(20)}$. Cuidar a una persona dependiente supone una notable fuente de estrés, que incrementa el riesgo de padecer diversos problemas físicos, así como importantes alteraciones emocionales, familiares y sociales ${ }^{(21)}$.

En el caso del cuidado de un niño con enfermedad crónica se produce un esfuer- 
zo físico, una limitación financiera, efectos emocionales y un aislamiento social que finalmente impacta la salud física y emocional del cuidador, porque este le da prioridad a las necesidades de su paciente sobre las propias, y toma las decisiones sobre esta base ${ }^{(22-24)}$. Esta situación puede llevar al cuidador a que desarrolle adicción al tabaco y/o al alcohol para poder cumplir las demandas del cuidado ${ }^{(25)}$.

Los cuidadores tienen miedo de ser testigos del sufrimiento y dolor de su familiar, a fracasar en su rol, a que las consecuencias negativas asociadas al cuidado afecten su forma de ser, a tomar decisiones equivocadas que lleven como consecuencia el malestar de los familiares. A la acumulación de estos miedos y preocupaciones, aunados a problemas de salud, psicológicos, emocionales, sociales y financieros, se le llama carga del cuidador ${ }^{(26)}$.

Para Zarit et al. ${ }^{(27)}$ la carga del cuidador, son las actitudes y reacciones emocionales ante la experiencia de cuidar y el grado de perturbaciones o cambios en diversos aspectos del ámbito doméstico y de la vida en general de los cuidadores. Este síndrome se desencadena por lo siguiente:

1. La transferencia afectiva de los problemas del enfermo hacia quienes le atienden.

2. La repetición de las situaciones conflictivas.

3. La sensación de percibirse abrumado por la carga del cuidado, lo que lleva al cuidador a la incapacidad para continuar con esta tarea.

La carga interfiere en el manejo adecuado del enfermo y en la propia evolución de la enfermedad; ya que disminuye la calidad de la atención que el cuidador brinda, impactando con esto en la adherencia al tratamiento y el pronóstico de la enfermedad ${ }^{(28-33)}$. Además de que puede ocasionar por un lado, episodios de mal- trato, tanto físico como psicológico hacia el enfermo ${ }^{(26)}$. Y por otro, el empeoramiento de la calidad de vida del cuidador $^{(10,24)}$.

Se ha demostrado que son numerosas las variables que inciden en el aumento de la carga del cuidador, de un lado están las relacionadas con el enfermo, como son el grado de deterioro y/o pérdida de autonomía. Por otro lado, las relaciones con el propio cuidador entre las que se encuentran la edad, el sexo, el estilo de afrontamiento, la motivación para el cuidado, las redes de apoyo y el tiempo de cuidado. Por último las derivadas de la relación afectiva cuidador-enfermo tanto previas como actuales ${ }^{(10,34,35)}$.

En esta dirección, el propósito del presente trabajo fue identificar las variables psicológicas y de características del cuidado que predicen la aparición de carga en cuidadores primarios informales de niños con cáncer que reciben atención médica en un hospital de tercer nivel en la ciudad de México.

\section{MÉTODO}

\section{Participantes}

La muestra estuvo integrada por 100 participantes, $93 \%$ eran mujeres y $7 \%$ hombres. La media de edad de los cuidadores fue de 35 años con una desviación típica de 9 años. Con respecto a la escolaridad, $28 \%$ de los participantes contaban con primaria, $35 \%$ con secundaria, $12 \%$ con nivel técnico, $17 \%$ con preparatoria y $8 \%$ con licenciatura. La distribución respecto a la ocupación mostró que eran: $71 \%$ amas de casa, 18\% empleados(as), $5 \%$ comerciantes, $4 \%$ profesionistas y $2 \%$ desempleados(as).

Por lo que respecta al estado civil fueron: $45 \%$ casados, $36 \%$ vivían en unión libre, 11\% divorciados(as), 6\% solteros(as) y $2 \%$ viudos(as). Y respecto al parentesco entre el cuidador y la persona a la que 
cuida, 91\% eran las madres, 5\% eran los padres y $4 \%$ eran otros familiares como abuelos y hermanos.

Las personas a las que cuidaban se distribuyeron de la siguiente forma: respecto al género eran $52 \%$ ) niñas y $48 \%$ niños. La edad osciló entre 4 meses a 18 años, con una media de 8 años y una desviación de 5. Y respecto al diagnóstico $35 \%$ tenían Sarcoma, 29\% Leucemia y 36\% tenían otro tipo de tumor.

\section{Instrumentos}

1. Formato de consentimiento informado (revisado y autorizado por el Comité de Ética de la institución).

2. Hoja de datos sociodemográficos. Como edad, género, ocupación, escolaridad, etc.

3. Encuesta de salud para el Cuidador Primario Informal ${ }^{(36)}$. Es un cuestionario diseñado para describir las características del cuidador informal, consta de 73 ítems organizados en cinco apartados: I. Características sociodemográficas del cuidador, II. Cuidado del paciente, III. Apoyo social percibido, IV. Prácticas preventivas de salud y V. Percepción de salud. Para fines de este estudio se utilizó sólo el apartado II.

4. Escala de carga del cuidador de Zarit. Se utilizó la versión validada en México por Montero, Jurado, Valencia, Méndez y Mora ${ }^{(37)}$. El análisis factorial exploratorio y confirmatorio de la escala mostró que está conformada por tres factores que explican el $50 \%$ de la varianza. Tuvo un alfa de Cronbach de 0,84., y el modelo tuvo un buen ajuste con valores iguales o superiores a 0,90 . Se trata de una escala autoaplicable conformada de 12 reactivos que proporcionan un puntaje de carga global e integran tres factores: impacto del cuidado, relación interpersonal y expectativas de autoeficacia. Para calificar la escala se sumaron los puntajes de todos los reactivos y se interpretó el nivel de carga dando el nivel de ausencia de carga a los puntajes de 1 a 22, carga leve de 23 a 29 y carga severa de 30 a 48 .

5. Inventario de Ansiedad de Beck. Es una escala autoaplicable de 21 reactivos. Se utilizó la versión validada con población mexicana por Robles, Varela, Jurado y $P_{a} z^{(38)}$. Esta versión demostró una consistencia interna de 0,83. La evaluación de la severidad de los síntomas se hace mediante una escala de 0 a 3 puntos, donde 0 indica poco o nada, 1 más o menos, 2 moderadamente y 3 severamente. Para calificar el instrumento se suman los puntajes obtenidos en cada uno de los reactivos y se interpreta el nivel de ansiedad de la siguiente manera: mínima de 1 a 5, leve de 6 a 15, moderada de 16 a 30 y severa de 31 a 63 puntos.

6. Inventario de Depresión de Beck II. El instrumento es autoaplicable y consta de 21 reactivos, cada uno con cuatro afirmaciones que describen el espectro de severidad de la categoría sintomática. La evaluación de los síntomas se hace mediante una escala de 0 a 3. Conforme aumenta el puntaje, es mayor la severidad del síntoma. Se utilizó la versión validada en México por Andrade ${ }^{(39)}$. Esta versión demostró una consistencia interna de 0,95. Para calificar el instrumento, se suman los puntajes obtenidos en cada uno de los reactivos y se interpreta el nivel de depresión de la siguiente manera: mínima de 1 a 4 , leve de 5 a 13 , moderada de 14 a 27 y severa de 28 a 63 puntos.

7. Escala de Modos de Afrontamiento al Estrés de Lazarus y Folkman. Fue diseñado para evaluar la forma en que los sujetos afrontan diversas situaciones generadoras de estrés. Se utilizó la versión validada con población mexicana por Marín y López ${ }^{(40)}$. Esta versión mostró un alfa de Cronbach global de 0,90. Fue una escala autoaplicable y estaba conformada de 57 reactivos que integran cinco factores denominados por el tipo de reactivo que conglomeran, estos se describen a continuación: afronta- 
miento dirigido al problema, pensamiento mágico, distanciamiento, búsqueda de apoyo social y dirigirse a lo positivo.

8. Escala de apoyo familiar para CPI. Evalúa la percepción de apoyo familiar que tienen los $\mathrm{CPI}$ de pacientes pediátricos con cáncer. Se construyó para población mexicana por Marín ${ }^{(41)}$. Tuvo un solo factor de 17 reactivos y mostró una confiabilidad de 0,95. La evaluación del nivel de apoyo percibido se hizo mediante una escala de 1 a 4 (nunca, casi nunca, casi siempre y siempre) y la puntuación final es de 17 a 68. Para calificar la escala se sumaron los puntajes de todos los reactivos y se interpretó el apoyo percibido dando el nivel de apoyo nulo a los puntajes de 17 a 51, apoyo mediano-bajo de 52 a 60, apoyo mediano-alto de 61 a 64 y apoyo altoadecuado de 65 a 68 .

\section{Procedimiento}

Se integró una batería formada por las escalas antes descritas. Posteriormente, se llevó a cabo la aplicación en el área de oncología de un hospital de tercer nivel en el sur del Distrito Federal en un periodo de tres meses. Se acudía a la cama del paciente durante el horario de visita, la investigadora se presentaba como psicóloga del área de Psico-oncología y se hablaba con la persona que estuviera acompañándolo sobre el lugar de origen, la enfermedad, el tiempo del diagnóstico, el tratamiento y si era el cuidador primario del paciente. Posteriormente, se les comentaba que se estaba realizando un estudio para saber cómo se sentían con la enfermedad y la hospitalización del paciente, se les invitaba a participar y se les preguntaba si sabían leer y escribir.

A los sujetos que afirmaban ser los cuidadores primarios del paciente y que querían participar en la investigación, se les leía el consentimiento informado, se les aclaraban dudas y se les pedía que lo firmaran. Posteriormente, se les explicaba cómo debían responder los instrumentos y se les pedía que lo hicieran en el transcurso de la mañana, cada hora se regresaba para responder dudas. Cada aplicación fue realizada en una sesión.

\section{Diseño}

De acuerdo con la clasificación sugerida por Kerlinger y Lee $^{(42)}$ se trata de un estudio no experimental, transversal.

\section{Análisis de datos}

Para identificar las variables que predicen la aparición de carga en los CPI de niños con cáncer se realizó un análisis de regresión múltiple por pasos, teniendo la carga global como variable dependiente y como predictores o variables independientes las variables psicológicas: ansiedad (los factores Subjetivo, Neurofisiológico, Autonómico y Pánico), depresión (los factores Afectivo-Cognoscitivo y Somático), los estilos de afrontamiento y apoyo familiar; y las características del cuidado: tiempo de cuidado del paciente, horas diarias de cuidado del paciente, número de actividades que realiza el CPI y número de áreas afectadas en la vida del CPI.

\section{RESULTADOS}

Se utilizó el paquete estadístico SPSS, versión 17.0 para Windows.

\section{Características del cuidado}

Se encontró que los participantes cuidaron a sus pacientes cuando estaban en casa por más de 9 horas diarias; el 70\%. Además, realizaron como parte de su rol de una a diez de las siguientes actividades: acompañar al paciente, bañarlo, alimentarlo, vestirlo, cargarlo, transportarlo, dar medicamentos, llevarlo al médico, com- 
prar medicinas y programar citas médicas ( $\bar{X}=8$ actividades, $\sigma=3$ actividades).

Los participantes han cuidado a sus pacientes a partir de su enfermedad de un mes a diez años ( $\bar{X}=18$ meses, $\sigma=22$ meses). Se exploraron las siguientes áreas de la vida del CPI para conocer si se habían afectado por asumir este rol: trabajo, escuela, tareas del hogar, vida social, vida familiar, vida sexual, pasatiempos, vacaciones y convivencia con la pareja. Se encontró que 10 participantes no tienen afectada ninguna área de su vida; mientras que los $90 \mathrm{CPI}$ restantes tenían afectadas de una a ocho áreas ( $\bar{X}=3$ áreas, $\sigma=2$ áreas).

Descripción de las variables psicológicas

Se encontró que el $74 \%$ de la muestra presentó algún nivel de carga; de éstos, el $28 \%$ en nivel excesivo. Tal como se muestra en la tabla 1.

Se encontró que toda la población presentó síntomas de ansiedad y depresión, los cuales aparecieron en el $16 \%$ de la población en un nivel severo en la primera variable y en el $20 \%$ de la segunda. Tal como se muestra en la tabla 2.

Con el fin de identificar los estilos de afrontamiento más frecuentes en la muestra se convirtieron las sumatorias de cada estilo de afrontamiento a puntajes $Z$ y con ellos se dividió a la población entre el grupo con los puntajes más altos y el grupo con los puntajes más bajos en cada estilo de afrontamiento, tomando como criterio de clasificación los percentiles 75 y 25 , respectivamente. $Y$ se encontró que los más frecuentes fueron los afrontamientos de pensamiento mágico y búsqueda de apoyo social. Tal como se muestra en la tabla 3.

Se encontró que la red de apoyo está integrada de una a 12 personas ( $\bar{X}=5$ familiares, $\sigma=2$ familiares). $Y$ el nivel de apoyo que perciben los $\mathrm{CPI}$ se ubica en un nivel medio bajo (32\%) o nulo (27\%). Tal como se muestra en la tabla 4.

\section{Tabla 1. Nivel de carga que presentan los CPI $(n=100)$}

\begin{tabular}{cc}
\hline Nivel & $\%$ \\
\hline Ausencia de carga & 26 \\
Carga leve & 46 \\
Carga excesiva & 28 \\
\hline
\end{tabular}

Media $=26,20$

Desviación Estandar=6,36

Tabla 2. Nivel de ansiedad y depresión que presentan los CPI $(\mathbf{n = 1 0 0 )}$

\begin{tabular}{ccc}
\hline \multirow{2}{*}{ Nivel } & Ansiedad & Depresión \\
\cline { 2 - 3 } & & Porcentaje \\
\hline Mínima & 20 & 6 \\
Leve & 34 & 28 \\
Moderada & 30 & 46 \\
Severa & 16 & 20 \\
\hline
\end{tabular}

Media $\mathrm{BAI}=17,61 ; \mathrm{BDI}=18,91$.

Desviación Estandar BAI=12,08; $\mathrm{BDI}=180,69$ 


\section{Tabla 3. Frecuencia de los estilos de afrontamiento más frecuentes que presentan los CPI de niños con cáncer $(n=100)$}

\begin{tabular}{cc}
\hline Estilos de afrontamiento & $\%$ \\
\hline Dirigido al problema & 26 \\
Pensamiento mágico & 31 \\
Distanciamiento & 29 \\
Búsqueda de apoyo social & 31 \\
Dirigirse a lo positivo & 27 \\
\hline
\end{tabular}

Tabla 4. Nivel de apoyo que presentan los CPI $(n=100)$

\begin{tabular}{cc}
\hline Nivel & \% \\
\hline Apoyo nulo & 28 \\
Apoyo medio bajo & 32 \\
Apoyo medio alto & 13 \\
Apoyo alto & 28 \\
\hline
\end{tabular}

Media $=56,26$

Desviación Estandar $=11,15$

Relación de las variables

sociodemográficas, médicas, psicológicas y las características del cuidado

Se realizó una correlación de Pearson para establecer la relación de la carga con las variables sociodemográficas, médicas, psicológicas y las características del cuidado; en todos los análisis sólo se retomaron las correlaciones estadísticamente significativas mayores a 0,30.

Se encontró una correlación positiva de la carga con el número de áreas afectadas en la vida del CPI $(r=0,37, p=0,01)$, con ansiedad $(r=0,47, p=0,01)$, con depresión $(r=0,47, p \leq 0,01)$ y una correlación negativa con el apoyo familiar $(r=-0,35, p=0,01)$. Mientras las demás variables no mostraron correlaciones estadísticamente significativas. Tal como se muestra en la tabla 5 .

Se realizó una correlación de Pearson para establecer la relación de ansiedad con las variables sociodemográficas, médicas, psicológicas y las características del cuidado. Se encontró una correlación posi- tiva entre ansiedad con depresión $(r=0,63$, $p=0,01)$. Y una correlación negativa con apoyo familiar $(r=-0,32, p=0,01)$. Mientras las demás variables no mostraron correlaciones estadísticamente significativas. Tal como se muestra en la tabla 6 .

Se realizó una correlación de Pearson para establecer la relación de depresión con las variables sociodemográficas, médicas, psicológicas y las características del cuidado. Se encontró una correlación negativa del apoyo familiar con la depresión. Mientras las demás variables no mostraron correlaciones estadísticamente significativas. Tal como se muestra en la tabla 7.

Se realizó una correlación de Pearson para establecer la relación de los estrategias de afrontamiento con las variables sociodemográficas, médicas, psicológicas y las características del cuidado. Se encontró una correlación positiva del estilo de afrontamiento dirigido al problema con el número de actividades que realiza el CPI $(r=0,31, p=0,01)$. Mientras las demás 
Tabla 5. Correlación de carga con las variables sociodemográficas, médicas, psicológicas y de características del cuidado $(n=100)$

Variables sociodemográficas, médicas, psicológicas y de características del cuidado

Edad del cuidador

Edad del paciente

Nivel socioeconómico

Número de hijos

Tiempo de diagnóstico de la enfermedad del paciente

Horas diarias de cuidado del paciente

Número de actividades que realiza el CPI

Número de áreas afectadas en la vida del $\mathrm{CPI}$

Ansiedad global

Factor Subjetivo de ansiedad

Factor Neurofisiológico de ansiedad

Factor Autonómico de ansiedad

Factor Pánico de ansiedad

Depresión global

Factor Afectivo-Cognoscitivo de depresión

Factor Somático de depresión

Afrontamiento global

Afrontamiento Dirigido al

problema

Afrontamiento Pensamiento mágico

Afrontamiento Distanciamiento

Afrontamiento Búsqueda de apoyo social

Afrontamiento Dirigirse a lo positivo

Apoyo familiar

Carga

global

$-0,125$

$-0,175$

$-0,066$

$-0,023$

0,065

$-0,018$

$-0,089$

$0,376^{* *}$

$0,475^{* *}$

$0,478^{* *}$

$0,427^{* *}$

$0,260^{* *}$

0,308**

$0,477^{* *}$

$0,429^{* *}$

$0,525^{* *}$

0,125

0,083

0,138

$0,201^{*}$

0,062

$-0,002$

$-0,352^{* *}$

** La correlación es significativa al nivel 0,01

* La correlación es significante al nivel 0,05

\section{Factores de carga}

Impacto del Relación Expe
cuidado interpersonal

Expectativas de autoeficacia

$-0,055$

$-0,126$

$-0,091$

$-0,095$

$-0,184$

$-0,088$

0,029

$-0,077$

$-0,133$

$-0,088$

0,070

0,008

0,057

$-0,069$

0,150

\section{0,054}

$-0,075$

$-0,053$

$$
0,011
$$

$-0,181$

$-0,045$

0,500**

$0,270^{* *}$

$-0,158$

$0,411^{* *}$

0,310 ${ }^{* *}$

$0,199^{*}$

0,381 ${ }^{* *}$

$0,299^{* *}$

$0,268^{* *}$

$0,392^{* *}$

$0,252^{*}$

0,172

0,168

$0,239^{*}$

0,127

0,394**

$0,229^{*}$

$-0,113$

0,360**

$0,306^{* *}$

$0,294^{\text {** }}$

$0,325^{* *}$

$0,273^{* *}$

$0,265^{* *}$

$0,408^{* *}$

$0,349^{* *}$

$0,291^{\text {** }}$

0,111

0,020

0,118

0,058

$-0,065$

0,193

0,122

0,045

0,104

0,149

$0,217^{*}$

0,030

0,078

0,042

$-0,016$

0,098 
Tabla 6. Correlación de ansiedad con las variables sociodemográficas, médicas, psicológicas y de características del cuidado $(n=100)$

\begin{tabular}{|c|c|c|c|c|c|}
\hline \multirow{2}{*}{$\begin{array}{l}\text { Variables } \\
\text { sociodemográficas, } \\
\text { médicas, psicológicas } \\
\text { y de características del } \\
\text { cuidado }\end{array}$} & \multirow{2}{*}{$\begin{array}{l}\text { Ansiedad } \\
\text { global }\end{array}$} & \multicolumn{4}{|c|}{ Factores de ansiedad } \\
\hline & & Subjetivo & Neurofisiológico & Autonómico & Pánico \\
\hline Edad del cuidador & $-0,235^{*}$ & $-0,295^{* *}$ & $-0,194$ & $-0,062$ & $-0,062$ \\
\hline Edad del paciente & $-0,174$ & $-0,223^{*}$ & $-0,099$ & $-0,106$ & $-0,063$ \\
\hline Nivel socioeconómico & $-0,062$ & $-0,065$ & $-0,062$ & $-0,118$ & 0,080 \\
\hline Número de hijos & 0,005 & $-0,015$ & $-0,019$ & 0,097 & 0,024 \\
\hline $\begin{array}{l}\text { Tiempo de diagnóstico } \\
\text { de la enfermedad del } \\
\text { paciente }\end{array}$ & $-0,017$ & $-0,045$ & 0,067 & $-0,129$ & 0,037 \\
\hline $\begin{array}{l}\text { Horas diarias de cuidado } \\
\text { del paciente }\end{array}$ & 0,036 & 0,039 & 0,027 & 0,049 & $-0,006$ \\
\hline $\begin{array}{l}\text { Número de actividades } \\
\text { que realiza el CPI }\end{array}$ & 0,031 & 0,039 & $-0,019$ & 0,012 & 0,110 \\
\hline $\begin{array}{l}\text { Número de áreas } \\
\text { afectadas en la vida del } \\
\text { CPI }\end{array}$ & $0,247^{*}$ & $0,198^{*}$ & $0,286^{* *}$ & 0,084 & $0,246^{*}$ \\
\hline Depresión global & $0,630^{* *}$ & $0,628^{* *}$ & $0,556^{* *}$ & $0,439^{* *}$ & $0,349^{* *}$ \\
\hline $\begin{array}{l}\text { Factor Afectivo- } \\
\text { Cognoscitivo de } \\
\text { depresión }\end{array}$ & $0,592^{* *}$ & $0,598^{* *}$ & $0,521^{* *}$ & $0,416^{* *}$ & $0,296^{* *}$ \\
\hline $\begin{array}{l}\text { Factor Somático de } \\
\text { depresión }\end{array}$ & $0,554^{* *}$ & $0,542^{* *}$ & $0,489^{* *}$ & $0,347^{* *}$ & $\mathbf{0 , 3 8 2}{ }^{* *}$ \\
\hline Afrontamiento global & $0,253^{*}$ & $0,250^{*}$ & 0,180 & 0,143 & $0,285^{* *}$ \\
\hline $\begin{array}{l}\text { Afrontamiento Dirigido } \\
\text { al problema }\end{array}$ & $0,242^{*}$ & $0,224^{*}$ & 0,167 & 0,152 & $0,317^{* *}$ \\
\hline $\begin{array}{l}\text { Afrontamiento } \\
\text { Pensamiento mágico }\end{array}$ & $0,338^{* *}$ & $0,366^{* *}$ & $0,266^{* *}$ & 0,196 & $0,206^{*}$ \\
\hline $\begin{array}{l}\text { Afrontamiento } \\
\text { Distanciamiento }\end{array}$ & $0,227^{*}$ & $0,244^{*}$ & 0,151 & 0,145 & 0,193 \\
\hline $\begin{array}{l}\text { Afrontamiento Búsqueda } \\
\text { de apoyo social }\end{array}$ & 0,109 & 0,084 & 0,104 & 0,052 & 0,154 \\
\hline $\begin{array}{l}\text { Afrontamiento Dirigirse } \\
\text { a lo positivo }\end{array}$ & 0,006 & 0,005 & $-0,042$ & $-0,034$ & 0,160 \\
\hline Apoyo familiar & $-0,324^{* *}$ & $-0,308^{* *}$ & $-0,375^{* *}$ & $-0,228^{*}$ & $-0,022$ \\
\hline
\end{tabular}

** La correlación es significativa al nivel 0,01

* La correlación es significante al nivel 0,05 
Tabla 7. Correlación de depresión con las variables sociodemográficas, médicas, psicológicas y de características del cuidado $(n=100)$

\begin{tabular}{lccc}
\hline \multirow{2}{*}{$\begin{array}{c}\text { Variables sociodemográficas, médicas, } \\
\text { psicológicas y de características del cuidado }\end{array}$} & $\begin{array}{c}\text { Depresión } \\
\text { global }\end{array}$ & \begin{tabular}{c} 
Factores de depresión \\
\cline { 3 - 4 } Cognoscitivo
\end{tabular} & Somático \\
\hline Edad del cuidador & $-0,163$ & $-0,170$ & $-0,159$ \\
Edad del paciente & $-0,058$ & $-0,103$ & $-0,043$ \\
Nivel socioeconómico & $-0,223^{*}$ & $-0,218^{*}$ & $-0,215^{*}$ \\
Número de hijos & 0,090 & 0,077 & 0,039 \\
Tiempo de diagnóstico de la enfermedad del & 0,014 & $-0,042$ & 0,106 \\
paciente & $-0,004$ & 0,058 & $-0,107$ \\
Horas diarias de cuidado del paciente & $-0,126$ & $-0,115$ & $-0,180$ \\
Número de actividades que realiza el CPI & 0,162 & 0,174 & 0,147 \\
Número de áreas afectadas en la vida del CPI & 0,122 & 0,112 & 0,070 \\
Afrontamiento global & 0,019 & 0,025 & $-0,026$ \\
Afrontamiento Dirigido al problema & $0,281^{* *}$ & $0,266^{* *}$ & $0,218^{*}$ \\
Afrontamiento Pensamiento mágico & 0,167 & 0,111 & 0,185 \\
Afrontamiento Distanciamiento & 0,051 & 0,070 & $-0,025$ \\
Afrontamiento Búsqueda de apoyo social & $-0,022$ & $-0,027$ & $-0,035$ \\
Afrontamiento Dirigirse a lo positivo & $\mathbf{- 0 , 4 6 7 ^ { * * }}$ & $\mathbf{- 0 , 5 0 1 * *}$ & $\mathbf{- 0 , 3 4 9 *}$ \\
Apoyo familiar & & & \\
\hline
\end{tabular}

** La correlación es significativa al nivel 00,01

* La correlación es significante al nivel 00,05

variables no mostraron correlaciones estadísticamente significativas. Tal como se muestra en la tabla 8.

\section{Predicción de la carga global}

Se realizó un análisis de regresión múltiple por pasos, teniendo la carga como variable dependiente y como predictores o variables independientes las variables psicológicas: ansiedad, depresión, los estilos de afrontamiento y apoyo familiar; y las características del cuidado: tiempo de cuidado del paciente, horas diarias de cuidado del paciente, número de actividades que realiza el CPI y número de áreas afectadas en la vida del CPI.

En el primer paso del análisis se incluyó la variable depresión como predictor de la carga. Los resultados de este primer paso, indican que la regresión fue significativa y que dicha variable predice de manera confiable la carga $(F 1=28.93, p=0,000)$, explicando el $22 \%$ de la varianza. En el segundo paso, se incluyó la variable número de áreas afectadas en la vida del CPI en la ecuación predictora; el incremento en la $R$ cuadrada fue a $R^{2}=0,319$, así como el cambio en $\mathrm{F}$ a F2=13,03, $p=0,000$, lo que indica que su contribución para explicar la carga fue relevante. Finalmente ingresó a la ecuación la variable ansiedad, el incremento en $\mathrm{R}$ cuadrada fue significativo $\left(R^{2}=0,348\right)$, así como el cambio en $\mathrm{F}$ $(F 3=4,26, p=0,042)$. En total las tres variables que resultaron ser predictores confiables de la carga explicaron el 34\% de la varianza. Tal como se observa en la tabla 9. 
Tabla 8. Correlación del afrontamiento con las variables sociodemográficas, médicas, psicológicas y de características del cuidado $(n=100)$

\begin{tabular}{|c|c|c|c|c|c|c|}
\hline \multirow{2}{*}{$\begin{array}{c}\text { Variables } \\
\text { sociodemográficas, } \\
\text { médicas, psicológicas } \\
\text { y de características del } \\
\text { cuidado } \\
\end{array}$} & \multirow[b]{2}{*}{$\begin{array}{l}\text { Afrontamiento } \\
\text { global }\end{array}$} & \multicolumn{5}{|c|}{ Estilos de afrontamiento } \\
\hline & & $\begin{array}{l}\text { Dirigido al } \\
\text { problema }\end{array}$ & $\begin{array}{l}\text { Pensamiento } \\
\text { mágico }\end{array}$ & Distanciamiento & $\begin{array}{c}\text { Búsqueda } \\
\text { de apoyo } \\
\text { social }\end{array}$ & $\begin{array}{c}\text { Dirigirse } \\
\text { a lo } \\
\text { positivo }\end{array}$ \\
\hline Edad del cuidador & 0,063 & 0,173 & 0,013 & $-0,154$ & 0,036 & 0,099 \\
\hline Edad del paciente & $-0,068$ & $-0,057$ & $-0,022$ & $-0,049$ & $-0,059$ & $-0,073$ \\
\hline Nivel socioeconómico & 0,019 & 0,094 & 0,015 & $-0,052$ & $-0,035$ & 0,010 \\
\hline $\begin{array}{l}\text { Número de hijos que } \\
\text { tiene el CPI }\end{array}$ & $-0,082$ & $-0,026$ & $-0,068$ & $-0,008$ & $-0,161$ & $-0,052$ \\
\hline $\begin{array}{l}\text { Tiempo de diagnóstico } \\
\text { de la enfermedad del } \\
\text { paciente }\end{array}$ & $-0,072$ & 0,018 & $-0,127$ & $-0,021$ & $-0,185$ & 0,036 \\
\hline $\begin{array}{l}\text { Horas diarias de } \\
\text { cuidado del paciente }\end{array}$ & $0,223^{*}$ & $0,254^{*}$ & 0,188 & $-0,034$ & $0,208^{*}$ & 0,170 \\
\hline $\begin{array}{l}\text { Número de actividades } \\
\text { que realiza el } \mathrm{CPI}\end{array}$ & $0,259^{* *}$ & $0,310^{* *}$ & 0,130 & 0,148 & 0,154 & 0,191 \\
\hline $\begin{array}{l}\text { Número de áreas } \\
\text { afectadas en la vida } \\
\text { del CPI }\end{array}$ & 0,049 & $-0,049$ & 0,067 & 0,115 & 0,112 & $-0,030$ \\
\hline Apoyo familiar & $0,218^{*}$ & $0,212^{*}$ & 0,081 & 0,161 & 0,171 & 0,190 \\
\hline
\end{tabular}

** La correlación es significativa al nivel 00,01

*La correlación es significante al nivel 00,05

Tabla 9. Modelos de predicción de la carga global del CPI $(n=100)$

\begin{tabular}{|c|c|c|c|c|c|c|}
\hline Modelo & Variable predictora & $\beta$ & Sig., & $\begin{array}{l}\text { Cambio } \\
\text { de la F }\end{array}$ & $\begin{array}{l}\text { Sig., del cambio } \\
\text { de la } F\end{array}$ & $\mathbf{R}^{2}$ \\
\hline 1 & Depresión & 0,477 & 0,000 & 28,93 & 0,000 & 0,228 \\
\hline \multirow[t]{2}{*}{2} & Depresión & 0,428 & 0,000 & 13,03 & 0,000 & 0,319 \\
\hline & $\begin{array}{c}\text { Número de áreas afectadas } \\
\text { en la vida del CPI }\end{array}$ & 0,306 & 0,000 & & & \\
\hline \multirow[t]{3}{*}{3} & Depresión & 0,292 & 0,007 & 4,26 & 0,042 & 0,348 \\
\hline & $\begin{array}{c}\text { Número de áreas afectadas } \\
\text { en la vida del CPI }\end{array}$ & 0,273 & 0,002 & & & \\
\hline & Ansiedad & 0,223 & 0,042 & & & \\
\hline
\end{tabular}

\section{DISCUSIÓN}

Los resultados encontrados confirmaron que los CPI de niños con cáncer en la mayoría de los casos de la ciudad de Mé- xico, es la madre tal como se ha observado en otros estudios ${ }^{(11,14,17,18,20,24,43-48)}$.

Posiblemente porque, la madre en general, cree que "nadie está a la altura para cuidar, proteger y atender las necesidades 
de su hijo" como ella lo hace ${ }^{(49,50)}$, así mismo, la maternidad es un componente principal de la identidad femenina que regula la vida de las mujeres; y aunque estas adoptan cada vez más roles fuera del hogar, la relación emocional con sus hijos sigue siendo fuerte y la abnegación maternal las coloca en primer lugar ${ }^{(51)}$.

La edad del CPI de la muestra se aproximó a lo encontrado en diferentes estudios realizados con cuidadores de niños con cáncer ${ }^{(9,18,24,33,35,44,46,48,50,52,53)}$. Son cuidadores en edad productiva a quienes se les suma la carga de atender a un hijo con un padecimiento crónico.

Todos los participantes han cuidado a sus pacientes desde el inicio de la enfermedad. Es decir, de un mes a diez años, con un promedio de 18 meses. El tiempo de cuidado se aproximó al reportado en otros estudios ${ }^{(24,50)}$. Cabe señalar que se ha encontrado con cuidadores de adultos que el tiempo de cuidado se relaciona con diversas implicaciones emocionales como carga, ansiedad, depresión y con la calidad de vida en general ${ }^{(54-56)}$. Sin embargo, en este estudio no resultaron significativas las correlaciones del tiempo de cuidado con estas variables; esto en coincidencia con Rubira et al. ${ }^{(24,50)}$.

Las discrepancias posiblemente se deban a que en cuidadores de niños con cáncer se ha observado esta relación con la calidad de vida y no con trastornos emocionales específicos como los que se evaluaron en el presente estudio ${ }^{(15,25,57-62)}$.

Las áreas que más se han afectado en la vida de los cuidadores de la muestra evaluada coinciden con el hecho de que la enfermedad del paciente impacta en la vida familiar, principalmente al aumentar las demandas del cuidador se alteran sus tareas domésticas y la relación que tiene con los otros hijos y con la pareja, lo cual ocasiona redistribución de roles, dificultades en la comunicación, alteraciones en las relaciones sexuales, distanciamien- tos e incluso infidelidades. Por otro lado, las exigencias de tiempo pueden obligar a los cuidadores a renunciar a su traba$\mathrm{jo}^{(3,15,24,60,63-68)}$.

Respecto a la carga se encontró que los datos de este estudio coinciden con lo reportado por otros autores respecto a que la mayoría de los participantes presentaron algún nivel de carga $a^{(1,15,18,24,33,48,49,50,58,59,74,87)}$.

Esto se debe a que por las características del cáncer los pacientes requieren mayor cuidado y los participantes se desgastan de forma permanente y continua por las constantes demandas que tienen por la ejecución de su rol, causando serios daños en la vida de los cuidadores ${ }^{(87,20)}$. La disminución de la calidad de vida y la carga del cuidador puede comprometer la atención que proporcionan a los pacientes, así como ocasionar desequilibrios en su salud ${ }^{(24)}$.

Por otro lado, toda la muestra presentó síntomas de ansiedad; sin embargo, sólo la minoría de los cuidadores mostró un nivel severo de ansiedad (16\%). Esto coincide con lo reportado por Martínez ${ }^{(44)}$ quien encontró que sólo el $17 \%$ de los cuidadores de niños con leucemia presentó un nivel severo. Sin embargo, nuestros resultados difieren respecto a lo reportado por $\operatorname{Ramos}^{(88)}$ donde sólo el $54 \%$ de los participantes mostraron ansiedad. Lo antes descrito, aporta evidencia que apoya la segunda hipótesis para esta fase del estudio respecto a que los $\mathrm{CPI}$ de niños con cáncer presentarían ansiedad.

Se ha reportado que los cuidadores que son generalmente más ansiosos antes de desempeñar su rol de cuidador son más propensos a experimentar mayores niveles de ansiedad y a desarrollar síntomas de estrés agudo ${ }^{(18,89)}$.

Respecto a depresión se observó, que toda la muestra presenta síntomas de depresión; sin embargo, sólo el $20 \%$ en un nivel severo. Esto coincide con lo reportado por Greening y Stoppelbein ${ }^{(90)}$ quienes encontraron que el $4 \%$ de la muestra te- 
nía un nivel severo. Martínez ${ }^{(44)}$ encontró síntomas depresivos en toda la población evaluada, pero sólo el 10\% mostró un nivel severo. Rubira et al. ${ }^{(50)}$ reportaron que sólo el $15 \%$ de la población evaluada presentó síntomas depresivos severos. Lobst et al. (26) encontraron que sólo el 9\% de la muestra tenía depresión severa y $\operatorname{Ramos}^{(88)}$ encontró que el $8 \%$ de la muestra tenía un nivel severo. Los cuidadores que presentan ansiedad y depresión durante el desempeño de su rol de cuidador, pueden tener un factor de vulnerabilidad para el desarrollo de un trastorno de estrés postraumático ${ }^{(30)}$.

Los resultados de este estudio mostraron una correlación positiva entre ansiedad y depresión. Esto coincide con lo reportado en población general ${ }^{(69,70)}$ y en cuidadores de niños con cáncer ${ }^{(44,46,48,71)}$. Además, se observó que existe una correlación de estas variables con carga, en plena coincidencia con lo informado en la literatu$\mathrm{ra}^{(1,14,24,33,53,72)}$. Por otro lado, las variables antes descritas mostraron una correlación negativa con apoyo familiar, coincidiendo con lo reportado en la literatura ${ }^{(14,24,59,73,74)}$

La depresión, la ansiedad y la carga son reacciones emocionales que surgen en el cuidador como consecuencia de la necesidad que tienen de adaptarse al inicio abrupto de la enfermedad, las dificultades para enfrentarla, la adaptación a una nueva rutina, ambientes y el miedo al empeoramiento del estado de salud del paciente ${ }^{(67)}$.

Las investigaciones realizadas sobre afrontamiento han encontrado que los cuidadores de niños con cáncer utilizan el afrontamiento enfocado al problema en un intento por modificar o eliminar las fuentes de estrés mediante la toma de decisiones y la acción directa al realizar ciertas conductas enfocadas al problema ${ }^{(91,93)}$. Mientras que el afrontamiento enfocado a regular la emoción es una respuesta cognoscitiva para analizar las consecuencias emocionales de los estresores y ayuda a mantener el equilibrio emocional al ser usado para alterar el significado de una situación y aumentar la percepción de control del individuo sobre sus estresores ${ }^{(92)}$.

Los resultados del presente estudio mostraron que los cuidadores utilizaron estilos de afrontamiento centrados tanto en el problema como en la emoción; destacando principalmente las estrategias de pensamiento mágico y búsqueda de apoyo social. Esto coincide con lo reportado por algunos autores que mencionan que los cuidadores utilizaron estrategias de afrontamientos mixtas ${ }^{(16,66,94-97)}$. Mientras que difiere en lo encontrado en otras investigaciones en donde los participantes sólo utilizaron estrategias dirigidas al problema $^{(52,98-101)}$.

Los cuidadores del presente estudio utilizan estrategias de afrontamiento mixtas que les permiten centrar sus acciones en el análisis o la valoración de las demandas o amenazas provocadas por la enfermedad de sus pacientes, para entenderla y poder crear planes de acción que les permita manejar la tensión provocada por la situación ${ }^{(101)}$.

Es decir, la enfermedad establece una serie de nuevas demandas que tienen que ser resueltas conociendo la enfermedad y su tratamiento; además, es necesario afrontar las emociones que surgen de lo que el cuidador piensa sobre esta y el futuro del paciente. En este contexto el afrontamiento enfocado al problema no está dirigido propiamente a resolver el problema curando al paciente porque esto no lo pueden hacer los cuidadores, sino a encontrar formas para manejar los síntomas que se experimentan por los efectos secundarios de la enfermedad. Mientras que, el afrontamiento enfocado a la emoción está dirigido a modificar los significados de la enfermedad del paciente, redefiniéndola como factor de crecimiento personal y familiar ${ }^{(16,99)}$.

Los estilos de afrontamiento que el cuidador utiliza están relacionados con su calidad de vida. Se ha observado que 
los participantes que tienen impacto en su calidad de vida por las implicaciones del cuidado utilizan estrategias de evitación o negación de la enfermedad; mientras que, los cuidadores que no ven afectada su calidad de vida utilizan estrategias racionales como diseñar un objetivo o aprender más de la enfermedad ${ }^{(24)}$.

Respecto al nivel de apoyo familiar que perciben los CPI se ubica en un nivel medio; esto coincide con lo reportado por un estudio sobre apoyo percibido comparando madres hispanas y madres anglosajonas, encontrado que las madres hispanas reportaron menores niveles de percepción de apoyo de la familia y los amigos ${ }^{(102)}$. Los resultados difieren de lo reportado por otros autores respecto a que la mayoría de la muestra evaluada percibía un nivel alto de apoyo, principalmente de la familia en general $^{(52)}$ o la pareja en particular ${ }^{(44) \text {. Lo }}$ antes descrito, aporta evidencia que refuta la cuarta hipótesis para esta fase del estudio respecto a que los $\mathrm{CPI}$ de niños con cáncer percibirían un nivel alto de apoyo familiar.

En la mayoría de los casos las fuentes de apoyo del cuidador son las creencias religiosas individuales, la familia, el equipo de salud y los amigos ${ }^{(94,95,97)}$. Los cuidadores demandan principalmente apoyo moral o emocional; es decir, necesitan sentirse comprendidos respecto a lo que les está sucediendo con su paciente enfermo ${ }^{(5,52,103,104)}$.

Durante el diagnóstico del cáncer el paciente pediátrico, la familia y en especial el cuidador recibe una enorme cantidad de apoyo familiar, social e institucional para lograr afrontar la crisis que representa la enfermedad y adaptarse a ella; el apoyo va disminuyendo durante el primer año del tratamiento y se estabiliza hasta después del quinto año de la enferme$\operatorname{dad}^{(16,67,97,100,102,105,106) \text {. }}$

Por otro lado, las variables que predijeron la carga fueron depresión, el número de áreas afectadas en la vida del CPI y ansiedad. Esto coincide con lo reportado por Norberg ${ }^{(74)}$ quien menciona que la influencia de la enfermedad en la vida cotidiana predice la carga. Rubira et al.(50) encontraron que la depresión es la variable que más varianza explica para predecir la carga en el cuidador. Y Rubira et al. ${ }^{(24)}$ reportaron que la salud general (ansiedad y depresión) y los costos adicionales debido a la función del papel del cuidador, entre otras variables predicen la carga en este.

La ansiedad y la depresión son las variables que aparecieron en el modelo de predicción de la carga debido a que el desconocimiento de una condición genera ansiedad ${ }^{(75)}$ y en los cuidadores el no tener conocimiento sobre la enfermedad y los procedimientos médicos que tienen que realizar para cuidar a sus pacientes les general ansiedad. Por otro lado, la falta de reconocimiento, reforzamiento o autoreforzamiento genera depresión ${ }^{(76)}$ y como los cuidadores no reciben remuneración económica ni reconocimiento porque su rol se ve como una obligación o continuidad lógica de las tareas domésticas. Asimismo la familia les aumenta la culpa por dejar a los otros hijos o al esposo y como ellos tampoco se auto-refuerzan puede aparecer la depresión.

La presencia de depresión y ansiedad en los CPI se puede explicar desde el enfoque cognitivo conductual ${ }^{(75-77)}$. La primera a partir de los tres conceptos que explica el sustrato psicológico de la misma: la triada cognitiva, los errores cognoscitivos (distorsiones cognitivas) y los esquemas. Es decir, los cuidadores presentan tres patrones cognitivos: tienen una visión negativa de sí mismos en cuanto al desempeño de su rol cuidador, interpretan las experiencias de la enfermedad de su paciente y el tratamiento de manera negativa y se tienen una visión negativa del futuro del pronóstico de la enfermedad del paciente.

Además presentan errores en el procesamiento de la información al realizar infe- 
rencias arbitrarias, generalización excesiva y maximizaciones o minimización ${ }^{(76)}$; es decir, distorsionan la información obtenida del equipo médico respecto al pronóstico de la enfermedad del paciente para que sus creencias puedan mantener su validez a pesar de que exista evidencia contraria.

Mientras que, las creencias centrales negativas que dominan en estos $\mathrm{CPI}$ son las que se asocian con el desamparo ${ }^{(78)}$; es decir, son ideas dominantes acerca de ellos mismos que surgen a partir del distrés psicológico que les ocasiona la enfermedad y el cuidado de su paciente y se identifican en los participantes a partir de frases como "soy incapaz de cuidar a mi hijo por eso enfermo" o "Soy un fracaso como mamá".

Al igual que en la depresión como se mencionó anteriormente, los CPI que presentan ansiedad también poseen ciertos errores en el pensamiento como catastrofización, abstracción selectiva y perdida de perspectiva, pensamiento automático y dicotómico ${ }^{(75,77)}$, es decir, distorsionan la información obtenida del equipo médico y de los otros cuidadores respecto al pronóstico de la enfermedad del paciente para para que sus creencias respecto al miedo que sienten por la situación amenazante pueda mantener su validez a pesar de que exista evidencia contraria.

Los hallazgos de la presente investigación permiten concluir que es necesario diseñar programas de intervención psicoterapéuticas que disminuyan la carga emocional que presentan los CPI y la vulnerabilidad que tienen para convertirse en pacientes potenciales; además, se ha demostrado que la calidad de vida de los pacientes pediátricos con cáncer se relaciona con el estado emocional de sus cuidadores $^{(79,80)}$. Po lo antes descrito, es necesario rescatar las recomendaciones de expertos internacionales respecto a la urgente necesidad de atender tanto a niños y adolescente con cáncer ${ }^{(81-84)}$ como a los integrantes de sus familias ${ }^{(3,85,86)}$.
Finalmente, se sugiere que en futuros estudios se utilice una muestra mayor que permita realizar un análisis de trayectoria utilizando el software AMOS para proponer un modelo que permita predecir la aparición de la carga en esta población.

\section{REFERENCIAS}

1. Aung L, Saw S, Chan M, Khaing T, Quah T, Verkooijen $\mathrm{H}$. The hidden impact of childhood cancer on the family: A multi-institutional study from Singapore Ann Acad Med Singapore 2012;41:170-5.

2. Cuesta C. Family care in chronic conditions: an approach to the literature. Colombia 2004; 13:137-46.

3. Grau C, Espada M. Percepciones de los padres de niños enfermos de cáncer sobre los cambios en las relaciones familiares. Psicooncología 2012;9:125-36. Doi: 10.5209/rev_psic.2012.v9.n1.39142

4. Brown R, Daly B, Rickel U. Enfermedades crónicas en niños y adolescentes. México: El Manual Moderno, 2008.

5. Castillo E, Chesla A, Echeverry G, Tascón E, Charry M, Chicangana J. Satisfacción de los cuidadores con la atención de adultos y niños con cáncer. Colomb Med 2005;36:43-9.

6. Coletto M, Camara S. Coping strategies and illness perception in parents of children with chronic diseases. Diversitas: Perspectiva en Psicología 2009;5:97-110.

7. Llorens, Mirapeix, López-Fando. Hermanos de niños con cáncer: resultado de una intervención psicoterapéutica. Psicooncología 2009;6:469-83.

8. Negre A, Fortes I. Programa de educación sanitaria: estrategias para disminuir la ansiedad que provoca la transición del hospital a casa, en los padres de niños con cáncer. Psicooncología 2005;2:157-62.

9. Cernvall M, Alaie I, Essen V. The factor structure of traumatic stress in parents of children with cancer: A longitudinal analysis. J Pediatr Psychol 2012;37:44857. Doi: 10.1093/jpepsy/jsr105 
10. Espín A. Caracterización psicosocial de cuidadores informales de adultos mayores con demencia. Rev Cub Salud Pública 2008:34. En línea. [Acceso el 15 de diciembre de 2011] Disponible en: http:// scielo.sld.cu/pdf/rcsp/v34n3/spu08308. Pdf

11. Holosbach G, Soares T. Experiencies of caregivers of children and teenagers with cáncer. Série: Ciências da Saúde 2010;11:89-108.

12. Vásquez B. La importancia del cuidador en los cuidados paliativos. Informe Médico 2006; 8:1-4.

13. Ramos R. Emergencia del cuidado informal como sistema de salud. México: Miguel Ángel Porrúa. 2008.

14. Klassen A, Raina P, Reineking S, Dix D, Pritchard S, O'Donnell M. Developing a literature base to understand the caregiving experience of parents of children with cancer: A systematic review of factors related to parental health and well-being. Support Care Cancer 2007;15:807-18. Doi: 10.1007/s00520-007-0243-x

15. Kazak A, Boeving C, Alderfer M, Hwang W, Reilly A. Posttraumatic stress symptoms during treatment in parents of children with cancer. J Clin Oncol 2005;23:740510. Doi.org/10.1200/JCO.2005.09.110

16. Clarke N, McCarthy M, Downie P, Ashley $\mathrm{D}$, Anderson V. Gender differences in the psychosocial experience of parents of children with cancer: A review of the literature. Psychooncology 2009;18:907-15. Doi:10.1002/pon.1515

17. Lafaurie M, Barón P, León S, Martínez M, Molina Q, Rojas C. Mothers caring for their children with cancer. Rev Colomb Enferm 2009;5:41-52.

18. Patiño-Fernandez A, Pai L, Alderfer M, Hwang T, Reilly A, Kazak A. Acute stress in parents of children newly diagnosed with cancer. Pediatr Blood Cancer 2008;50:289-92.

19. López A, Blanco D. La importancia de un buen cuidador: guía para cuidadores de personas dependientes. España: Gobierno de La Rioja, 2006.

20. Medeiros B, Baena L. Caregivers of children with cancer: Aspects of life affected by the caregiver role. Revista Brasileña de Enfermería 2007a; 60:670-5.

21. Crespo M, López J. El apoyo de los cuidadores de mayores dependientes: presentación del programa "cómo mantener su bienestar". Informaciones Psiquiátricas 2007; 188:1-15.

22. Lawrence V, Murray J, Samsi K, Banerjee S. Attitudes and support needs of Black Caribbean, South Asian and White British careers of people with dementia in the UK. Br J Psychiatry 2008;193:240-6. Doi: 10.1192/bjp.bp.107.045187

23. Meyler E, Guerin S, Kiernan G, Breatnach F. Review of family-based psychosocial interventions for childhood cancer. J Pediatr Psychol 2010;35:1116-32. Doi. org/10.1093/jpepsy/jsq032

24. Rubira E, Marcon S, Belasco A, Gaíva M, Espinosa M. Burden and quality of life of caregivers of children and adolescents with chemotherapy treatment for cancer. Acta Paulista de Enfermagem 2012; 25:567-73.

25. Litzelman K, Catrine K, Gangnon R, Witt W. Quality of life among parents of children with cancer or brain tumors: the impact of child characteristics and parental psychosocial factors. Qual Life Res 2011;20:1261-9. Doi.org/10.1007/ s11136-011-9854-2

26. Lobst E, Alderfer M, Jane $O$, Askins M, Fairclough D, Katz E, Butler R, Dolgin M, Noll R. Problem solving and maternal distress at the time of a child's diagnosis of cancer in two-parent versus lone-parent households. J Pediatr Psychol 2009;34:817-21.

27. Zarit S, Reever K, Bach-Peterson J. Relatives of the impaired elderly: Ccorrelates of feeling of burden. Gerontologist 1980;20:64955. Doi: 10.1093/geront/20.6.649

28. Dahlquist M, Shroff P. When distraction fails: parental anxiety and children's re- 
sponses to distraction during cancer procedures. J Pediatr Psychol 2005;37:107688. Doi: 10.1093/jpepsy/jsi048

29. Matziou V, Perdikaris P, Feloni D, Moschovi M, Tsoumakas K, Merkouris A.Cancer in childhood: children's and parents' aspects for quality of life. Eur J Oncol Nurs 2008; 12:209-16. Doi: 10.1016/j. ejon.2007.10.005

30. Morris A, Gabert-Quillen C, Douglas D. The association between parent PTSD/Depression symptoms and child PTSD symptoms: A meta-analysis. J Pediatr Psychol 2012;37:1076-88. Doi:10.1093/jpepsy/ jss091

31. Power T. Stress and coping in childhood: The parent's role. Parenting: Science and Practice 2004;4:275-321. Doi:10.1207/ s15327922par0404_1

32. Robinson K, Gerhardt C, Vannatta K, Noll R. Parent and family factors associated with child adjustment to pediatric cancer. J Pediatr Psychol 2006;32:4000-410. Doi. org/10.1093/jpepsy/jsI038

33. Rodríguez M, Dunn M, Zuckerman T, Vannatta K, Gerhardt A, Compas B. Cancerrelated sources of stress for children with cancer and their parents. J Pediatr Psychol 2012; 37:185-97.

34. Duncan D, Tremont G. Impact of frontal systems behavioral functioning in dementia on caregiver burden. J Neuropsychiatry Clin Neurosci 2007; 19:43-9.

35. Yağc-Küpeli B, Akyüz C, Küpeli S, Büyükpamukçu M. Health-related quality of life in pediatric cancer survivors: A multifactorial assessment including parental factors. J Pediatr Hematol Oncol 2012; 34:194-9. Doi:10.1097/MPH.0b013e3182467f5f

36. Ramos R, Barcelata J, Alpuche R, Islas $S$, Salgado G. Evaluación diagnóstica del síndrome de burnout y entrenamiento para el manejo del estrés en cuidadores primarios informales de enfermos crónicos degenerativos. México: Facultad de Estudios Superiores Zaragoza/ Universidad Nacional Autónoma de México. 2006.
37. Montero X, Jurado S, Valencia A, Méndez J, Mora I. Escala de carga del cuidador de Zarit: evidencia de validez en México. Psicooncología 2014;11:73-86. Doi: 10.5209/rev_psic.2014.v11.n1.44918

38. Robles R, Varela R, Jurado S, Páez F. Versión mexicana del Inventario de ansiedad de Beck: propiedades psicométricas. Revista Mexicana de Psicología 2001; 18.221-218.

39. Andrade P. Propiedades psicométricas del inventario de depresión de Beck II en residentes de la ciudad de México. Tesis de Licenciatura no publicada. México: Facultad de Psicología/ Universidad Nacional Autónoma de México. 2010.

40. Marín Z, López S. Revisión de las propiedades psicométricas de la escala de afrontamiento de Lazarus y Folkman. Tesis de Licenciatura no publicada. México: Facultad de Psicología/ Universidad Nacional Autónoma de México. 2012.

41. Marín M. Desarrollo y validación de la escala de apoyo familiar en cuidadores del paciente pediátrico. Tesis de Maestría no publicada. México: Facultad de Psicología/ Universidad Nacional Autónoma de México. 2006.

42. Kerlinger F, Lee H. Investigación del Comportamiento. Métodos de investigación en ciencias sociales. México: McGraw-Hill, 2002.

43. Martínez G, Robles R, Ramos R, Santiesteban M, García V, Morales E, García L. Carga percibida del cuidador primario del paciente con parálisis cerebral infantil severa del Centro de Rehabilitación Infantil teletón. Revista Mexicana de Medicina Física y Rehabilitación 2008; 20:23-9.

44. Martínez P. Sintomatología de ansiedad, depresión y enojo en padres de niños con leucemia. Tesis de Licenciatura no publicada. México: Facultad de Psicología/ Universidad Nacional Autónoma de México. 2009.

45. Medeiros B, Baena L. Caregivers role strain in caregivers of children with cancer. Rev Bras Enferm 2007b; 60:513-518. 
46. Ramos B. Ansiedad y depresión en madres con hijos hospitalizados. Tesis de Licenciatura no publicada. México: Facultad de Psicología/ Universidad Nacional Autónoma de México. 2009.

47. Stam H, Grootenhuis M, Brons P, Caron $H$, Last B. Health-related quality of life in children and emotional reactions of parents following completion of cancer treatment. Pediatr Blood Cancer 2006; 47:3129. Doi:10.1002/pbc.20661

48. Steele R, Long A, Reddy K, Luhr M, Phipps S. Changes in parental distress and child-rearing strategies across treatment for pediatric cancer. J Pediatr Psychol 2003;28:447-52.

49. Beck A, López M. Tensão devido ao papel de cuidador entre cuidadores de criança com câncer. Rev Bras Enferm 2007; 60:513-8. Doi: 10.1590/S003471672007000500006

50. Rubira E, Munhoz G, Martínez E, Barbosa D, Silva A. Taking care of children with cáncer: Evaluation of the caregiver's burden and quality of life. Rev Latinoam Enferm 2011;19;515-22.

51. Young B, Dixon-Woods $M$, Findlay $M$, Heney D. Parenting in a crisis: Conceptualising mothers of children with cancer. Soc Sci Med 2002;55:1835-47. Doi. 10.1016/ s0277-9536(01)00318-5

52. Almunia A. Factores psicológicos y sociales en las neoplasias malignas pediátricas. Rev Cub Pediatría 2007:79. En línea. [Acceso el 15 de diciembre de 2011] Disponible en : http://scielo.sld.cu/scielo.php?pid=S0034$75312007000100001 \&$ script=sci_arttext

53. Pollock E, Litzelman K, Wisk L, Witt W. Correlates of physiological and psychological stress among parents of childhood cancer and brain tumor survivors. Acad Pediatr 2013; 13:1-10. Doi.org/10.1016/j. acap.2012.11.005

54. Amendola F, Oliveira M, Alvarenga M. Quality of life of family caregivers of patients dependent on the family health program. Texto \& Contexto Enfermage 2008; 17:266-72.
55. Cerda B, Díaz A, Lejia E, Díaz A. Evaluación de la psicopatología y calidad de vida en una muestra de cuidadores informales primarios de pacientes con esquizofrenia. Psiquis 2009; 18:35-43.

56. Martínez-Martín P, Rodríguez-Blázquez C, Forjaz M. Quality of life and burden in caregivers for patients with Parkinson's disease: Concepts, assessment and related factors. Expert Rev Pharm Out 2012; 12:221-30.

57. Chien L, Lo L, Chen C, Chen Y, Chiang C, Yu C. Quality of life among primary caregivers of Taiwanese children with brain tumor. Cancer Nurs 2003; 26:305-11. Doi.org/10.1097/00002820-20030800000009

58. Klassen A, Klaassen R, Dix D, Pritchard S, Yanofsky R, O'Donnell M. Impact of caring for a child with cancer on parents' health-related quality of life. I Clin Oncol 2008; 26:5884-9.

59. Klassen A, Raina P, McIntosh C, Sung L, Klaassen R, O'Donnell M, Yanofsky $R$, Dix D. Parents of children with cancer: which factors explain differences in health-related quality of life. Inter J Cancer 2011;129:1190-8. Doi.org/10.1002/ ijc. 25737

60. Pereira M, Magni R, Mingione I, Caran E. Os desafíos diários do cuidador de crianças e de adolescentes com câncer. Psicología, Teoría y Práctica 2009; 11:51-3.

61. Witt W, Litzelman K, Wisk L, Spear H, Catrine K, Levin N, Gottlieb C. Stress-mediated quality of life outcomes in parents of childhood cancer and brain tumor survivors: A case-control study. Qual Life Res 2010;19:995-1005. Doi:10.1007/s11136010-9666-9

62. Yamazaki S, Sokejima S, Mizoue T, Eboshida A, Fukuhara S. Health-related quality of life of mothers of children with leukemia in Japan. Qual Life Res 2005; 14:1079-85.

63. Baider L. Cáncer y familia: aspectos teóricos y terapéuticos. Inter J Clin Health Psychol 2003;3:505-20. 
64. Chacón-Muñoz D, Cisneros-Castolo M. Modelo de intervención AFASINCA para familiares de niños con cáncer. Revista de Enfermería del Instituto Mexicano del Seguro Social 2011;19:155-61.

65. López G, Pérez A. Afrontamiento de la familia en el proceso salud-enfermedad en niños con leucemia. Tesis de Licenciatura no publicada. México: Facultad de Estudios Superiores-Zaragoza/ Universidad Nacional Autónoma de México. 2004.

66. Moral R, Martínez R. Reacción ante el diagnóstico de cáncer en un hijo: estrés y afrontamiento. Psicol Salud 2009; 19:189-196.

67. Noel G. Participación de la familia en la adaptación de niños y adolescentes que sobreviven al cáncer. Anales de la Universidad Metropolitana 2011;11:71-89.

68. Pedro E, Funghetto S. Concepções de cuidado para os cuidadores: um estudo com a criança hospitalizada com câncer. Revista Gaúcha de Enfermagem 2005;26:210-9.

69. Naragon-Gainey K. Meta-analysis of the relations of anxiety sensitivity to the depressive and anxiety disorders. Psychol Bull 2010;136:128-50. Doi:10.1037/ a0018055

70. Stein D, Hollander E. Tratado de los trastornos de ansiedad. Barcelona: Psiquiatría, 2004.

71. Reeb B, Conger K, Wu Y. Paternal depressive symptoms and adolescent functioning: The moderating effect of gender and father hostility. Fathering 2010;8:131-42. Doi:10.3149/fth.0801.131

72. Mullins L, Wolfe C, Chaney M, Elkin D, Wiener L, Hullmann E, Fedele A, Junghans A. The relationship between single-parent status and parenting capacities in mothers of youth with chronic health conditions: The mediating role of income. J Pediatr Psychol 2011; 36:249-57. Doi:10.1093/ jpepsy/jsq080

73. Nájera G, Ledezma M, Patiño M, Galicia A. Incertidumbre en padres de niños con cáncer. Enfermería Neurológica 2010; 9:20-22.
74. Norberg A. Parents of children surviving a brain tumor: burnout and the perceived disease-related influence on everyday life. J Pediatr Hematol Oncol 2010; 32:285-9. Doi: 10.1097/MPH.0b013e3181e7dda6

75. Beck A, Emery G, Greenberg R. Anxiety disorders and phobias: a Cognitive Perspective. New York: Basic Books, 1985.

76. Beck A, Rush A, Shaw B, Emery G. Terapia cognitiva de la depresión. Bilbao: Desclée de Brouwer, 2008.

77. Greenberger D, Padesky C. El control de tú estado de ánimo. Barcelona: Paidós, 2010.

78. Beck J. Terapia Cognitiva: conceptos básicos y profundización. Barcelona: Gedisa, 2000 .

79. Castellano C, Sánchez T, Blasco T, Gros L, Capdevila L, Pérez-Campdepadrós M. Afrontamiento y malestar emocional parental en relación en la calidad de vida del adolescente oncológico en remisión. Psicooncología 2010;7:415-31.

80. Gonzalez-Arratia N, Nieto D, Valdez J. Resiliencia en madres e hijos con cáncer. Psicooncología 2011;8:113-23. Doi: 10.5209/rev_psic.2011.v8.n1.9

81. Bragado C. Funcionamiento psicosocial e intervenciones psicológicas en niños con cáncer. Psicooncología 2009;6:327-41.

82. Celma A. Psicooncología infantil y adolescente. Psicooncología 2009;6:285-290.

83. Orgilés M, Méndez F, Espada J. Prendimientos psicológicos pera el afrontamiento del dolor en niños con cáncer. Psicooncología 2009;6:343-56.

84. Méndez X, Orgilés M, López-Roing S, Espada J. Atención psicológica en el cáncer infantil. Psicooncología 2004;1:139-54.

85. García N, Day C. Family partnership model as a framewoek to address psychosocial needs in pediatric cáncer patients. Psicooncología 2009;6:357-72.

86. Alameda A, Barbero J. El duelo en padres del niño oncológico. Psicooncología 2009;6:485-98

87. Hutchinson K, Willard V, Hardy K, Bonner M. Adjustment of caregivers of pe- 
diatric patients with brain tumors: A cross-sectional analysis. Psychooncology 2009;18:515-23. Doi: 10.1002/pon.1421.

88. Ramos B. Ansiedad y depresión en madres con hijos hospitalizados. Tesis de Licenciatura no publicada. México: Facultad de Psicología/ Universidad Nacional Autónoma de México. 2009.

89. Alfaro R, Morales V, Vázquez P, Sánchez R, Ramos R. Guevara L. Sobrecarga, ansiedad y depresión en cuidadores primarios de pacientes con dolor crónico y terminales. Revista Médica del Instituto Mexicano del Seguro Social 2008; 46:485-494.

90. Greening L, Stoppelbein L. Brief Report: Pediatric cancer, parental coping style, and risk for depressive, posttraumatic stress, and anxiety symptoms. J Pediatr Psychol 2007; 32:1272-7.

91. Lazarus R, Folkman S. Stress, and coping. New York: Springer, 1984.

92. Lazarus R, Folkman, S. An analysis of coping in a middle-aged community simple. Journal of Health and Social Behavior 1980;21:219-39.

93. Pearlin L, Schooler C. The structure of coping. J Health Soc Behav 1978; 19:2-21.

94. Beltrao M, Vasconcelos M, Pontes C, Albuquerque M. Childhood cancer: Maternal perceptions and strategies for coping with diagnosis. J Pediatr (Rio J);2007;83:562-6. Doi: 10.2223/JPED.1723

95. Duarte C, Zanini N, Nedel B. O cotidiano dos pais de crianças com câncer e hospitalizadas. Revista Gaúcha de Enfermagem 2012; 33:111-118.

96. García R, B. Perfil del cuidador primario, sus estilos de afrontamiento y el vínculo afectivo con el enfermo oncológico infantil. Tesis de doctorado no publicada. España. Unversidad Ramon Llull/Facultad de Psicología. 2012.

97. Oliveira P, Schwartz E, Machado B, Burille A, Vestena Z, Machado F. Social network and support bonds of the families of children with cancer. Florianópolis 2010; 19:334-42.

98. Chacín F, Chacín J. Estrategias de afrontamiento en padres de niños y adolescentes con cáncer. Revista Venezolana de Oncología 2011; 23:199-208.

99. Espada M, Grau C. Estrategias de afrontamiento en padres de niños con cáncer. Psicooncología 2012; 9:25-40.

100. Hoekstra J, Jaspers J, Kamps W, Klip C. Psychological adaptation and social support of parents of pediatric cancer patients. J Pediatr Psychol 2011; 26:225-35.

101. Huerta H, Corona M, Méndez V. Evaluación de los estilos de afrontamiento en cuidadores primarios de niños con cáncer. Revista Neurología, Neurocirugía y Psiquiatría 2006; 39:46-51.

102. Wijnberg W, Kamps W, Klip C, Hoekstra W. Psychological distress and the impact of social support on fathers and mothers of pediatric cancer patients. J Pediatr Psychol 2006;31:785-792.

103. Cacante J, Arias V. Tocar los corazones en busca de apoyo: el caso de las familias de los niños con cáncer. Investigación y Educación en Enfermería 2009; 27:170-80.

104. Martínez F, Martínez J, Corbalán J. Cáncer infantil/adolescente: una aproximación psicológica. En J. Ortigosa M, Quiles y F. Méndez (Edits.), Manual de psicología de la salud con niños, adolescentes y familia. España: Pirámide. 2003.

105. Da Silva P, Galvao C, Melo R, Castanheira N. Apoyo social y familiar de niños con cáncer: revisión integradora. Revista Latinoamericana de Enfermería 2008; 16. En línea. [Acceso el 15 de diciembre de 2011]. Disponible en http:// www.scielo.br/scielo.php?pid=S010411692008000300023 \&script $=$ sci $_{\text {_ }}$ arttext\&tlng=es

106. Rosaleen O. Burden of care and childhood cancer: experiences of parents in Asian context. Health Soc Work 2003;28:232-40. 